\title{
Modelling of system impairments due to Four Wave Mixing in Dispersion Shifted links having non uniform zero-dispersion wavelength
}

\author{
Ernesto Ciaramella \\ CSELT- Centro Studi E Laboratori Telecomunicazioni \\ Via G.Reiss Romoli, 274 (10148) Torino ITALY \\ Phone: +39112285442 Fax +39112285840 \\ e-mail: ernesto.ciaramella@cselt.it
}

\begin{abstract}
.
Four Wave Mixing impairments in geographical WDM networks strongly depend on the zero dispersion wavelength of the fibre, that in actual links is far from being uniform. Here the role of non-uniform chromatic dispersion is investigated for the first time, using a new simplified and effective method. Obtained results show a decrease of the system penalty for increased fluctuations. However this is not a monotonic behavior, as resonance peaks can be observed. Monte Carlo simulations are also carried out, that give for the first time the estimation of the probability distribution function of the system penalty.
\end{abstract}

\section{INTRODUCTION}

Presently, it is widely accepted that optical networks would exploit Wavelength Division Multiplexing (WDM) technique. However, when implementing a WDM network, particular care should be taken in order to avoid the occurrence of a non linear propagation effect, namely the Four Wave Mixing (FWM). Indeed FWM could 
dramatically affect system performance, whenever the chromatic dispersion coefficient of the fibre is very low (Tkach, 1995); this is not the case of most of the regional and national fiber networks, that are typically made of conventional single mode fibre (G.652), having a non-negligible chromatic dispersion coefficient $(D=17$ $\mathrm{ps} / \mathrm{nm} / \mathrm{km}$ ). Actually, FWM has to be considered only in Dispersion Shifted (DS) fibers (G.653), which are usually deployed in the long international links, and also, in some countries, in the national transport network. Even in those fibers, FWM limitations strongly depend on the chromatic dispersion, or the zero-dispersion wavelength $\left(\lambda_{0}\right)$, of the fiber, so that, in principle, system impairments could be strongly reduced by a slight detuning from the optimum phase matching condition. In a uniform fiber this could be accomplished by setting the channels far enough from the $\lambda_{0}$ (Kurtze, 1994). However installed DS links are actually far from being uniform. Indeed they are made of different jointed spans, and the $\lambda_{0}$ value of those spans is randomly distributed according to the manufacturer statistical distribution. Thus the chromatic dispersion properties of those links can not be determined. Although FWM relevance can not be simply estimated in non-uniform fibers, FWM limitations over a given link are expected to be quite different from what would be observed in the corresponding uniform fiber case. Moreover, when considering a whole DS fiber network, FWM impairments should be rather investigated using a statistical approach, using Monte Carlo simulations. Unfortunately, a thorough analysis of the relevance of chromatic dispersion non-uniformity still lacks, mainly because it would have required a large amount of simulations, and those would have been much too time consuming if carried out by means of the usual numerical approach (Agrawal, 1995).

Here we present a new method for determining system penalties in WDM channels in non-uniform fiber links. This approach is used to evaluate FWM impairments in links with non-uniform chromatic dispersion. First, we considered a given link, and we adiabatically scaled the fluctuation magnitude: this alters FWM efficiency, but it does not monotonically affect the system penalties. Indeed, resonance peaks are found, arising from the partial phase matching produced in different spans. Occurrence of these peaks is observed even for relevant standard deviation of the $\lambda_{0}$. Monte Carlo simulations are also carried out, that give a quantitative estimation of the probability density function of the system penalty. 


\section{MODEL AND RESULTS}

Due to the relevant computing time of the standard method (Agrawal, 1995), it was necessary to implement a new, simplified tool in order to fast evaluate the eye closure of the channels affected by FWM. To this aim we developed a simplified analytical approach. For the sake of simplicity, we did not consider in-line amplifiers, and assumed all parallel polarisations, that maximise the FWM impairments. We also concentrated on partially degenerate FWM, also known as Three Wave Mixing (Wu, 1995, Cappellini 1991). It should be noted that this is likely the first relevant parametric process in WDM systems; indeed, in actual systems either the polarisations and the frequencies of the carriers do exhibit random fluctuations, thus a three wave coupling is far more likely to occur than the completely non-degenerate FWM.

Under the previous assumptions, system impairments were calculated using this tool. The method is based on the analytic solution of the coupled mode equations (Cappellini, 1991), neglecting the (low) energy transfer from the three signals to other frequencies. Although this approach requires much shorter computing time (by a factor $10^{3}$ ), tool results show very good agreement with those obtained by means of the most proper split-step simulations (Agrawal, 1995). This can be seen in Figure 1, reporting comparative results for the eye closure dependence on $D$ in uniform links (continuous curve: tool; circles: standard split-step simulations).

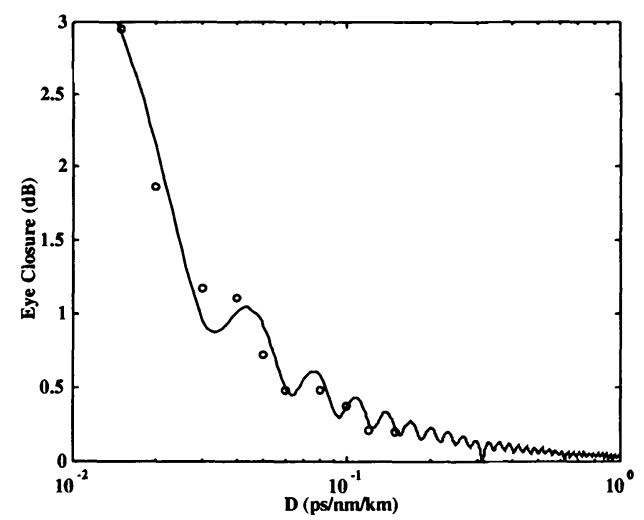

Figure 1 Comparison of the analytical tool results (line) and those obtained using a standard split-step method (circles). Results refer to the uniform case. 
This tool was used to estimate the FWM penalty in non-uniform DS links for the first time. We considered links composed of $3 \mathrm{~km}$ long uniform spans and with 0.24 $\mathrm{dB} / \mathrm{km}$ loss coefficient. Calculations were stopped to $30 \mathrm{~km}$ length, as after this distance propagation is almost linear, because of the fibre attenuation (in this case the non linear effective length would be less than $20 \mathrm{~km}$ ). The $\lambda_{0}$ 's were randomly chosen according to a gaussian distribution with $\left\langle\lambda_{0}>\right.$ and $\sigma$ parameters (respectively, the average zero dispersion wavelength and the standard deviation). Thus the chromatic dispersion at the signal wavelength $\lambda_{s}$ was calculated by the usual

$$
D\left(\lambda_{S}\right)=S\left(\lambda_{S}-\lambda_{0}\right)
$$

where $S$ is the dispersion slope $\left(S=0.07 \mathrm{ps} / \mathrm{nm}^{2} / \mathrm{km}\right.$ ). The effect of the fluctuations was first modelled by considering a simplified case with adiabatic switching from the uniform fibre. A set of ten $\Delta \tilde{\lambda}$ was sorted, according to a normal Gaussian distribution. The $\lambda_{0}$ 's were calculated, by scaling according to:

$\lambda_{0}=\lambda_{0}+\sigma(\Delta \tilde{\lambda})$. 


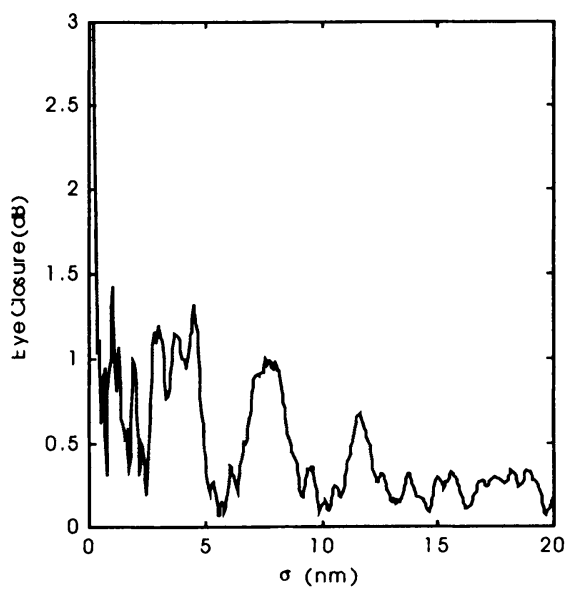

(a)

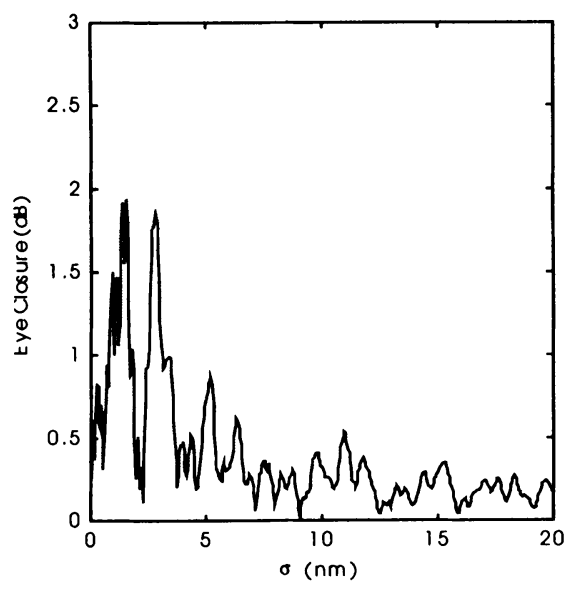

(c)

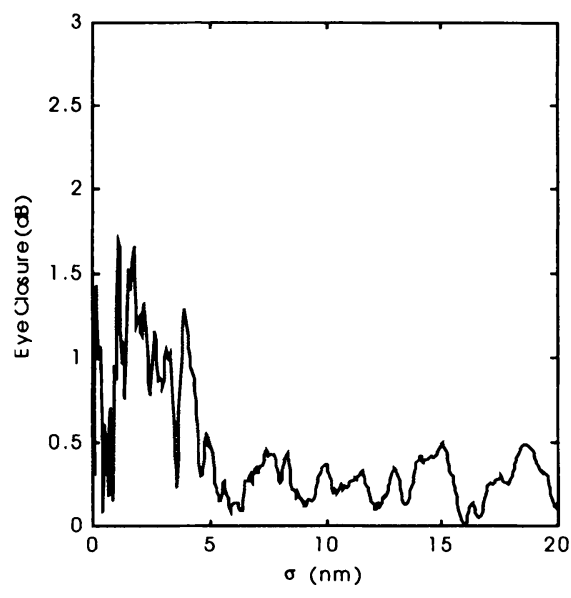

(b)

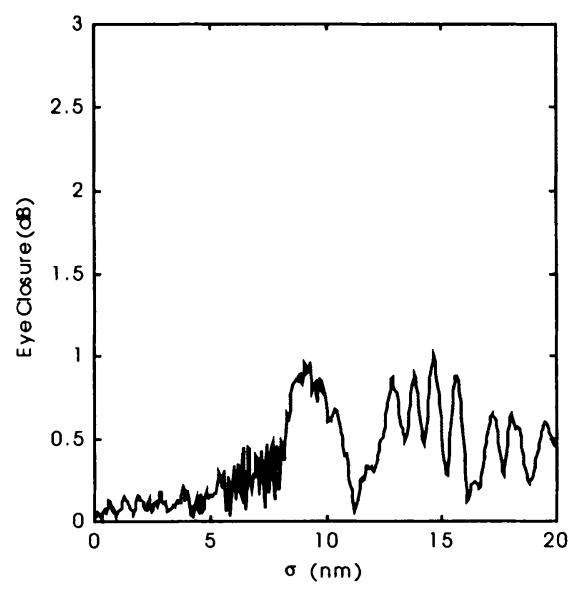

(d)

Figure 2 Effect of adiabatic increase of the zero dispersion wavelength fluctuations.

The four figures report the eye closure dependence on $\sigma$. The average chromatic dispersion coefficients are respectively 0 (a), 0.1 (b), 0.2 (c) and $1 \mathrm{ps} / \mathrm{nm} / \mathrm{km}$ (d). The WDM channels are equally spaced by $400 \mathrm{GHz}$.

According to Equation 2, the fluctuations were adiabatically switched on starting from $\sigma=0$ (uniform case) up to $20 \mathrm{~nm}$ (quite higher than observed values), with $0.1 \mathrm{~nm}$ step. For every $\sigma$ value the eye penalty was evaluated for the three interacting channels of a WDM system with $400 \mathrm{GHz}$ channel spacing and $4 \mathrm{~mW} /$ channel optical power. Figure 3 shows the typical results obtained by scaling the $\sigma$ value. In all the figures we report 
only the eye closure penalty referred to the central channel, which is typically the most affected. It is expected that fluctuations can mitigate the system impairments, with respect to the uniform case. This effect can be observed in Figure 2.a, that indeed refers to the average chromatic dispersion value $\langle\mathrm{D}\rangle=0 \mathrm{ps} / \mathrm{nm} / \mathrm{km}$.

Here for $\sigma=0 \mathrm{~nm}$ perfect phase matching occurs, giving a quite high penalty (more than $3 \mathrm{~dB}$ eye closure). When fluctuations increase phase matching is affected, and the penalty decreases. It is noteworthy, the effect over the system is clearly not monotonic; notable resonance peaks are indeed obtained, resulting from partial phase matching.

On the other hand, when we set a non-zero average dispersion value, different behaviours were obtained (see Figures 2.b and 2.c). Again, the fluctuation effect decreases the FWM impact, but here the phenomenon is less significant. Iindeed still for large fluctuations $(\sigma>10 \mathrm{~nm}) \mathrm{FWM}$ can originate system penalties not much lower than in the corresponding uniform case $(\sigma=0 \mathrm{~nm})$. Moreover, we found a quite surprising result for a still higher $\langle\mathrm{D}\rangle$ value (Figure 2.d). Here chromatic dispersion fluctuations make the FWM more effective and many penalty peaks can be observed for $\sigma \approx 10-15 \mathrm{~nm}$. Clearly, the behaviours shown in Figure 2 are rather general, but details of the curves are actually dependent on the initial $\Delta \tilde{\lambda}$ set.

In order to obtain a more quantitative assessment of the fluctuation relevance with respect to the uniform worst case, Monte Carlo simulations were eventually performed assuming $\sigma=5 \mathrm{~nm}$ (a realistic value) and $\langle D\rangle=0 \mathrm{ps} / \mathrm{nm} / \mathrm{km}$. System penalties were calculated over statistical ensembles of 200 DS fibre links, and an estimation of the probability distribution function was derived. Figure 3 shows the obtained histogram. The worst case penalty (more than $3 \mathrm{~dB}$ ) has negligible penalty, but high penalty values $(>1 \mathrm{~dB})$ are still likely. On the other hand, there is also a significant probability that fluctuations can decrease the penalty to negligible values $(<0.2 \mathrm{~dB})$. 


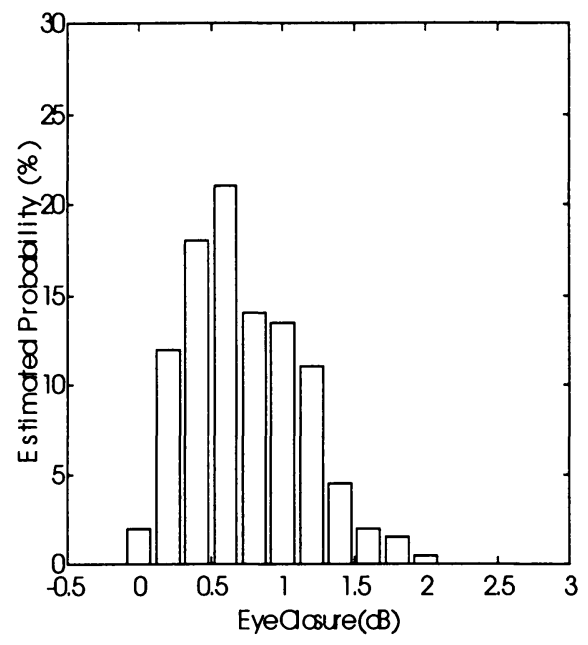

Figure 3 Probability histograms of the eye closure penalty for $\mathrm{P}=4 \mathrm{~mW} / \mathrm{channel}$. The average chromatic dispersion coefficient is $\langle\mathrm{D}\rangle=0 \mathrm{ps} / \mathrm{nm} / \mathrm{km}$ and the standard deviation is $\sigma=5 \mathrm{~nm}$.

\section{CONCLUSIONS}

We investigated the role of FWM in WDM systems in non-uniform DS links, by means of a new, effective tool, considering three signal interaction. It was demonstrated for the first time how the chromatic dispersion fluctuations can modify FWM limitations with respect to the uniform fiber case. Fluctuations generally diminish the impairments with respect to the worst case (corresponding to perfect phase matching), but resonance peaks can be produced by partial phase matching. This effect is confirmed by Monte Carlo simulations, showing a quite broad probability distribution function of the FWM penalty in a realistic DS fiber network. Obtained results indicate a statistical reduction of the penalty. Although in some cases there is a quite complete suppression of the nonlinear effect, FWM can still produce relevant system impairments.

\section{REFERENCES}

Agrawal G. P, Nonlinear fiber optics, Academic Press, 1995. 
110 Part Five: Transmission in optical transport networks II

Cappellini, G., Trillo S., "Third order three wave mixing in single mode fibres: exact solution and spatial instabilities", J. Opt. Soc. Am. B, 8, 824-838 (1991)

Fukui M., Aisawa S., Ishida O., Shimano K., Umeda A., Sakamoto T., Oda K., Takachio N. , "Allowable fibre input power for installed dispersion shifted fibre in equal/unequal channel spaced WDM transmission systems”, El. Lett.. 33, 639-640 (1997).

Kurtze C., "Suppression of fiber nonlinearities by appropriate dispersion management", IEEE Photonic Technol. Lett., 5, 1250-1253 (1993).

Tkach R., Chraplyvy A.R., Forghieri F., Gnauck A.H., Derosier R.M., "Four Photon Mixing and high speed WDM systems”, J. Lightwave Technol., 13, $841-849$ (1995)

Wu W., Yeh P., "Energy coupling by partially degenerate Four Wave Mixing in multichannel lightwave systems", Photon. Technol. Lett., 7, 545 (1995). 